\title{
Association between Sense of Loneliness, Social support, Quality of Life and Multimorbidity in Elderly Patients
}

\author{
Anna Vespa ( $\square$ a.vespa@inrca.it ) \\ INRCA-IRCCS https://orcid.org/0000-0002-0503-7852 \\ Roberta Spatuzzi \\ Azienda Ospedaliera Regionale San Carlo \\ Pisana Gattafoni \\ INRCA-IRCCS \\ Paolo Fabbietti \\ INRCA-IRCCS \\ Maria Velia Giulietti \\ INRCA-IRCCS
}

Research article

Keywords: Multimorbidity, Sense of loneliness, Quality of life, Elderly

Posted Date: July 21st, 2020

DOl: https://doi.org/10.21203/rs.3.rs-39487/v1

License: (9) This work is licensed under a Creative Commons Attribution 4.0 International License.

Read Full License 


\section{Abstract}

Multi-morbidity has been associated with adverse health outcomes, such as reduced physical function, poor quality-of-life(QoL), poor self-rated health. The adverse impact of multi-morbidity on other health domains is further exacerbated by other factors. In this study the association between quality of life, social support, sense of loneliness and sex, age in elderly patients affected by two or more chronic diseases (multi- morbidity) was evaluated. Patients n. 216 affected by tumors hypertension, cardiovascolar diseases, ictus, diabetes, all self-sufficient and living with family members. Tests: MMSEMini-Mental-State-Examination; ADL-Activities of Daily Living; Social-Schedule: demographic variables; Loneliness Scale -de Jong Gierveld; Quality-of-Life - FACT-G; WHOQOL-BRIEF social relationships. Statistical analysis: Multivariate Regression Analysis. The patients with three or more diseases have worse dimensions of QoL Physical-well-being $(p=.003)$, Social well-being $(p=.003)$, Emotional-wellbeing $(p=.012)$, Functional-well-being $(p<.001)$, than those with two. Multiple linear regression. QoL: PWB, SWB, EWB,FWB as dependent variables.In the presence of multi-morbidity with an increase in the patient's age PWB $(B=-.131, p=.014)$, SWB $(B=-.170, p=.009)$ EWB $(B=-.457, p<.000)$, FWB $(B=-.139, p$ $=.013)$, decreases by an average of 0.1 , and as the sense of solitude increases $P W B(B=-.425, p<.000)$, $\operatorname{SWB}(B=-.464, p<.000), E W B(B=-.457, p<.000), F W B(B=-468, p<.000)$ decrease by 0.4 . The sense of loneliness and increasing age are associated with bad quality-of life in self-sufficient elderly patients with multi-morbidity. Demonstrating that loneliness, also in presence of interpersonal relations, predicted worse quality of life helps identify people most at risk for common symptoms and lays the groundwork for research about diagnosis and treatment.

\section{Introduction}

Multimorbidity is a major concern for health services, health research and health

$(1-5)$. Defined as two or more long-term chronic conditions, multimorbidity has been described as the most common chronic condition experienced by adults (2). As a result of increasing longevity, multiple comorbid conditions, commonly referred to as 'multimorbidity', have also become progressively more common among older adults $(6,1,6-13)$. Moreover every published study describes the strong association of multimorbidity with age and that age is the main risk factor for prevalent and incident multi-morbidity $(1,8,9,14,15,16)$.

The present study will examine what affects emotional state and quality of life (Health Related Quality of Life -HRQoL), when two or more physical illnesses are present in an elderly person and whether the sense of loneliness plays a role in this regard. Although more attention has recently been paid to this phenomenon $(17,18)$ there are still many gaps in our knowledge. In fact, relatively little is known about the association between physical multimorbidity and loneliness $(6,19,20)$.

At this point it is necessary to distinguish the social support, from the sense loneliness that can manifest itself even in the presence of significant relationships $(21,22)$. Furthermore, loneliness and life alone are 
related but not overlapping categories. Studies on older adults show that living alone is not necessarily indicative of loneliness $(23,24)$ : people living alone reporting frequent social contacts and active social involvement in community organizations (20). Thus, researchers have distinguished loneliness from the experience of being alone or solitary. The latter reflects a state of social isolation that implies a voluntary departure from one's social network, while solitude is involuntary and more closely associated with deficits in the perceived quality of one's social interactions (23). So by solitude we mean the discrepancy between actual and desired social relationships, (25) and explains the role of poor quality connections. Social isolation means an objective state of minimal social contact with other individuals, while solitude reflects a subjective state of lack of the desired affection and closeness to a significant other or intimate (eg, emotional loneliness ) or close friends and family members (eg relational solitude) and the sense of loneliness can also occur in the presence of family relationships.

As many study showed loneliness and depression are associated, in particular in older adults $(14,26,27)$.

Loneliness has been shown to prospectively predict increased depressive symptomatology, impaired cognitive performance, dementia progression, significant likelihood of nursing home admission, and multiple disease outcomes with functional limitations in elderly people (e.g., hypertension, heart disease, and stroke in older persons) $(1,6,7,17,27-31)$. So decreased physical and mental quality of life and early mortality are among others associated with loneliness particularly among the elderly $(15,21,25$, 32-35).

So far, studies linking physical illness and solitude and/or sense of loneliness are relatively few in particular on the association between physical multimorbidity and solitude and / or sense of solitude. $(36,37)$. Furthermore, as far as we know, the few studies undertaken so far on the association between the number of diseases / multimorbidity and loneliness or vice versa have been limited to middle-aged and older adults (age $\geq 45$ ) and have produced conflicting results $(22,34,38,39)$. A multimorbidity study between men and women aged 45 and over in Canada and Australia shows that multimorbidity was significantly linked to loneliness in all age groups, with the exception of Australian women aged 75 and older (40).

Regarding social networks, even less studies exist. While longitudinal results are lacking, it has been reported that individuals with four or more chronic illnesses are more likely to have limited social networks compared with individuals with one or less chronic conditions.(41). Cross- sectional studies have shown that physical multimorbidity is associated with an increased risk of loneliness $(29,42)$. Moreover some studies provide a longitudinal view of the relationship between loneliness and multimorbidity, but rather than assessing the impact of multimorbidity on loneliness over time, they investigate how the number of chronic conditions affects loneliness (22). Some authors in one of their studies (19) empathized the complex nature of the relationship of multimorbidity with loneliness and social network size, and affirm that little is known about the underlying mechanisms so far, and further researches on relationship quality and the subjective perception of one's social network and their relationship with loneliness and multimorbidity are of great interest. Other authors suggest that 
materialist models of multimorbidity and functional limitation at older age cannot, on their own, explain the health inequalities as the behavioural and psycho-social factors play an important role $(6,15,27,30$, 31,43 ). Given the limited number of research on the association between (physical) multimorbidity and loneliness and the sense of loneliness, and the mixed findings from research that has focused on the multimorbidity-loneliness association in middle-aged and older adults, this study had the following objectives: (1) to examine the association between physical multimorbidity and loneliness in elderly population sample; and (2) to determine if any factors might be important for this association. To this end, we examine sense of loneliness (SoL) in presence of interpersonal relationships; association and effects on the health of SoL as potential mechanisms underlying the association between SoL and HRQoL and health among vulnerable elderly individuals with multi-morbidity. This analysis may suggest intervention strategies to alleviate loneliness and to face on the disease conditions in old age.

\section{Method}

This study uses data from the Multidimensional Evaluation Form in Geriatrics project, a prospective observational study aimed at collecting data about the patterns and quality of prescriptions among older patients admitted to acute care wards of geriatric medicine located in Central Italy. Multimorbidity is defined as the simultaneous presence of two or more chronic physical health conditions. For this analysis, some chronic health conditions were included, namely tumors, cardiovascular diseases, chronic pulmonary diseases, diabetes mellitus, hypertension, stroke. All these conditions, were assessed by diagnosis of the disease carried out by the doctors. Additionally, the use of treatment/medication received in the 12 months prior to interview was indicative of a diagnosis and was included in prevalence estimates for each disease.

\section{Participants}

Two hundred fifty tree patients affected by different diseases were consecutively recruited and asked to participate. during follow-up medical visits at the Departments of INRCA, National Institute of Science and Health for Aging. Only two hundreds fourteen patients joined the study. Fifty two did not meet the inclusion criteria or did not answer all the questionnaires: it was therefore decided not to consider them in the study. So the sample included n. 162 Patients. The demographic variables are described in Table 1. They compiled the expected tests administered by specifically trained psychologists. The diagnosed diseases by the physicians were the following: tumors, cardiovascular diseases, diabetes, hypertension, ictus, pulmonary diseases. The presence of two or more pathologies in a single patient has been documented by the physicians (Table 2). Moreover the patients showed a medium (7\%) to good (93\%) self- functional sufficiency and intact cognitive abilities evaluated respectively by the following tests: ADL (Activities of Daily Living) and MMSE.

After completing the initial medical examination they were referred to the investigator. Inclusion criteria included age (over 65), with diagnosis of different diseases (from 1 to 4 years); presence of social relations. Patients were excluded if: they refused to participate; were unable to provide informed consent; 
had other forms of disease (dementia, terminal illness); MMSE > 24; were not self-sufficient; were using any type of psychotropic drugs (including antidepressants); they have no social relations.

Age and demographic data including marital status and educational levels were collected (Tab.1). No one of the examined subjects had financial difficulties.

\section{Measures}

All the patients filled in the following tests:

a) $A D L$-Physical functioning: activities of daily living. Limitation in ADL was used to assess physical functioning $(44,45)$.

The questions were based on self- reported difficulty in engaging in activities during the last 30 days, using a five-point response scale ranging from none to extreme difficulty. The ADL measure included in SAGE was based on WHODAS 2.0 and has been validated in LMICs by WHO and collaborating agencies. WHODAS 2.0 is validated cross-culturally through a systematic research study. The cross-cultural applicability research study used various qualitative methods to explore the nature and practice of health status assessment in different cultures.

The study included linguistic analysis of health-related terminology, key informant interviews, focus groups, and quasi-quantitative methods such as pile sorting and concept mapping (carried out in tandem). Information was gathered on the conceptualization of disability and on important areas of dayto-day functioning. In this study, severe and extreme difficulties were combined to represent limitation in a particular activity. We have used an extended set of ADL that included sitting for long periods, walking $100 \mathrm{~m}$, standing up, standing for long periods, climbing one flight of stairs, stooping/kneeling/crouching, picking up things with fingers, extending arms above shoulders, concentrating for $10 \mathrm{~min}$, walking a long distance $(1 \mathrm{~km})$, bathing, getting dressed, carrying things, moving around inside home, getting up from lying down, and getting to and using the toilet. For the analysis, a dichotomous variable was created, which took value 1 if the respondent noted a limitation in one or more of the above ADLs (1+ ADL) and 0 otherwise.

b. MMSE-Mini Mental State Examination $(46,47)$ measures cognitive functions. The Italian versions of the MMSE modified from the Los Angeles Epidemiologic Catchment Area study was used. MMSE scores were dichotomized to indicate presence or absence of cognitive impairment using the published cut point of $\geq 24$ (cognitive impairment absent) and $<24$ (cognitive impairment present).

c. Social Schedule describing sex, age, marital status, educational levels, presence of different kinds of interpersonal relation, and a self-assessment of health by the patient. It consisted of a single question: "How would you classify your health: excellent, good, acceptable, bad or very bad?" The score for "very bad" was 1 and for "excellent" it was 5 . The patient was invited to select the most appropriate score. A schedule of clinical diagnosis (with years from first diagnosis) was included by physicians. 
d. Quality of Life -Functional-Assessment of Cancer-Therapy-General (FACT-G) (48) has all requirements including reliability and validity for use in oncology clinical trials (Italian version). The score sums up to a total ranging from zero to 108 points, where a higher score indicates better quality of life. It includes the following subscales: Physical-Well-Being (PWB), Social-Well-Being(SWB), Emotional-Well-Being(EWB), Functional-Well-Being(FWB), FACT-General-Summary-Score (FACT_G). The score sums up to a total ranging from zero to 108 points, where a higher score indicates better quality of life.

e. Loneliness Scale by Jenny de Jong Gierveld (49) consisting of 11 items; six are formulated negatively and five are formulated positively. Loneliness is seen as a subjective experience and is, as such, not directly related to situational factors. The scale describing the sense of loneliness, or subjective social isolation, is defined as a situation experienced by the participant as one where there is an unpleasant or inadmissible lack of (quality of) certain relationships. Loneliness includes situations where the number of existing relationships is smaller than desirable or acceptable, as well as situations where the intimacy wished for has not been realized. The11-items multidimensional scale describes the following dimensions : (1) severe feelings of loneliness as well as less intense loneliness feelings; (2) negative as well as positive items; and (3) a latent continuum of deprivation. In addition, the scale met the criteria of the dichotomous logistic Rasch model.

f. The questionnaire WHOQOL-BRIEF of social support (50. De Girolamo et al., 2000) was used. The validated Italian version was administered. It comprises 13 questions. This tool, consistent with its original concept, is self-administered. In case of an inability to comprehend any question, assistance was available as follows: the researcher read such part of the research slowly, using the same words in order to maintain exactly the same meaning.

\section{Clinical evaluation}

The different diagnosis were made by the physicians for each diseases following the guide lines for each pathologies and in the occasion of follow-up visits at the time of tests administration expected for this research.

\section{Statistical analysis}

Data were expressed as means \pm standard deviation (continuous variables) or as percentage (categorical variables) (Table1). The reliability of the FACT $-G$ subscales was assessed by Cronbach's coefficient alpha. The pathologies number was dichotomized by less than three and three or more pathologies. Statistical comparison between the these two categories of pathologies (multi-morbidity) and the dimensions of quality of life was performed by t-Student test. Moreover the sense of loneliness was categorize in four groups (not loneliness, moderate loneliness, severe loneliness, very severe loneliness). Moreover the WHOQOL was dichotomize by no or few social relations and presence of good social relations. Pearson's coefficient was used to assess correlations between studied variables. The multiple linear regression models was assessed to evaluate the associations between the dimensions of quality of life FACT_G and multimorbidity considering the effect of age, sex, sense of loneliness and WHOQOL. The 
significance was accepted for $p<0.05$. All analyses were performed using SPSS V19.0 Statistical Software Package for Windows.

\section{Results}

All the patients studied lived with relatives and have different relation with family members and friends. The presence of two or more pathologies in a single patient has been documented (Table 1). Moreover the patients showed a medium (7\%) to good (93\%) self-functional sufficiency evaluated by ADL (Activities of Daily Living).

We have taken into consideration the FACT quality of life scale The subscales are reliable: Physical-WellBeing (PWB) (Crombach's Alpha= 0.927), Social-Well-Being(SWB) (Crombach's Alpha= 0.927), EmotionalWell-Being(EWB) (Crombach's Alpha= 0.926), Functional-Well-Being(FWB) (Crombach's Alpha $=0.927)$ are reliable.

So we have considered these subscales of quality of life (FACT) for our analysis.

At first we evaluated whether significant differences emerged in these dimensions of quality of life by comparing patients with different chronic pathologies. No differences in quality of life dimensions between different pathologies emerged but only in presence of multimorbidity comparing two with tree or more pathologies. In fact significant differences in the dimensions of quality of life PWB ( $F=3.074$, $p=.003)$, SWB $(F=.341, p=.003)$, EWB $(F=1.360, p=.012), F W B(F=5.954, p<.001)$ emerged from the comparison between patients with two diseases and those with three and more than three pathologies. To be affected from three or more chronic diseases creates a negative blend of the dimensions of quality of life PWB, SWB, EWB and FWB (Table 2). It means that the quality of life decreases in elderly patients affected by three or more diseases, while the elderly patients with two pathologies maintain a good quality of life, considering that we have studied self-sufficient and with intact cognitive abilities patients.

Then we analyzed which conditions are associated with a sense of loneliness as subjective perception of relationships, and social support (perceived) (and demographic variables and how much these factors may be considered as cofactors in addition to the multimorbility in influencing the quality of life of these patients.

So we proceeded to the elimination of possible multicollinearity of the variables (the study of correlations), from which the following variables emerged as variables to be included in the model: Physical well-being, Social well-being, Emotional well-being, Functional well-being, sense of loneliness, presence of social relationships, age, sex, multimorbidity. The only variable not resulting in the model is the educational level.

\section{Results: Multiple linear regression}

At this point it has finally come to the multiple linear regression models where the dimensions of quality of life FACT_G- PWB, SWB, EWB and FWB, were considered as dependent variables for multimorbidity 
predictor also considering the correction for age, sex, sense of loneliness (4 categories), social support (2category WHOQOL) (Tab.2).

The linear regression model with dependent variable PWB has a good adjusted R-square (0.640). It emerges that in the presence of multimorbidity with an increase in the patient's age $(B=-.131, p=.014)$, the Physical well-being (PWB), decreases by an average of 0.1 , and as the sense of solitude increases $(B=$ $-.425, \mathrm{p}<.000)$, the PWB decreases by 0.4 . Instead, as good social relationships decrease, the PWB decreases by $0.4(B=463, p<.000)(T a b .3)$.

The linear regression model with Social Well-Being (SWB) dependent variable has a not very high adapted R-square (0.474). It emerges that in the presence of multimorbidity, the SWB decreases by an average of 0.1 , and as the sense of solitude increases, the SWB decreases by 0.4 . Instead, as good social relationships decrease, the SWB decreases by 0.3 .

It emerges that with an increase of the multimorbidity $(B=-.170, p=.009)$ SWB decreases by an average of 0.1 , and as the sense of solitude increases $(B=-.464, p<.000)$, the Social well-being decreases by 0.4 . Instead, as good social relationships decrease, the SWB decreases by $0.4(B=299, p<.001)$. (Table 4).

The linear regression model with Emotional Well-Being (EWB) dependent variable has a adapted R-square (0.628). EWB decreases by an average of 0.1 . It emerges that as the sense of solitude increases $(B=-.457$, $p<.000)$, the EWB decreases by 0.4 . Instead, as feelings of good social relationships decrease, the EWB decreases by $0.4(B=413, p<.000)$. (Table5).

The linear regression model with FWB dependent variable has an acceptable adjusted R-square (0.604). It turns out that in the presence of multimorbidity $(B=.-137, p=.012)$ the FWB decreases by 0.1 . Moreover when the patient's age increases $(B=-.139, p=.013)$ the FWB decreases by 0.1 . As the sense of loneliness increases $(B=-468, p<.000)$, the FWB decreases by 0.4 , while as as feelings of good social support decrease $(B=-388, p<.000)$ the FWB decrease by 0.3. (Table 6).

\section{Discussion}

These findings indicated that having a positive perception of supportive social environment increases the quality of life of elderly people with physical illnesses also in presence of chronic disease and multimorbidity. The dimensions PWB SWB EWB FWB of QoL increase in the absence of sense of loneliness in patients with multimorbidity The elderly person with chronic diseases maintains psycho-physical wellbeing even in the presence of multimorbidity if he does not feel alone.

The subjective perception of social isolation is considered as a risk of bad quality of life in patients being diagnosed with chronic illnesses and multimorbidity in presence of self-sufficiency and intact cognitive abilities The presence of a sense of loneliness is associated with bad PWB SWB EWB and FWB That is people with greater social participation and a positive feelings of relations have lower risk of a bad quality of life also in condition of suffering from multiple chronic diseases Some authors affirm that 
social isolation increases the risk of being diagnosed with chronic illnesses: people with greater social participation have lower risk of suffering from multiple chronic diseases Cantarero-Prieto 2018 This conclusion may be in agreement with some suggestion emerged from our results

We agree with the consideration of Cantarero-Prieto who affirms: "the risk linked to isolation sense of loneliness in presence of relations together with the traditional one associated with lifestyles should be considered in the development of new public policies in facing on the diseases especially in elderly people" Cantarero-Prieto 2018 The social relationships should be considered a fundamental aspect of an individual's lifestyle

Our results add a further consideration: it is not mere social isolation as an objective dimension but feeling lonely even in the presence of significant relationships that influence the psycho-physical health of the elderly.

On the basis of these results geriatric health professionals are encouraged to evaluate social relationships as subjective perception and stimulate support given by relatives friends or neighbors of elderly affected by diseases as dimension of good or bad quality of life ( Hero 2017). So our results showed the importance of subjective perception of quality of social relationships for promoting mental health and protecting against the development and progression of physical diseases: social relationships both quantity and quality are a major contributing factor in lowering broad-based morbidity and mortality. By contrast loneliness considered as the perceived absence of positive and meaningful social relationships has been linked to diminished quality of life in older individuals with multimorbidity also in presence of relations from a quantitative point of view.

Age was another factor emerged from our study: PWB and FWB decreases with increasing age This result confirms the results of other studies Salisbury 2011; Peters,2019).

Although most literatures agree that HRQoL decreases with increasing numbers of chronic diseases and with age, some studies have shown that the effects of multimorbidity on quality of life were not simply the sum of the effects of two or multiple diseases, or increasing age, but had different effects in combining different diseases (Walker, 2007; Persissinotto, 2016). This is perhaps because HRQoL was related to the individual life goals, expectations and concerns. This implies that the concept of HRQoL is subjective and multidimensional, including positive and negative elements (Bao, 2019). In addition, with increasing disease, people seemed to get used to and adapt to their disease with the consequence that they subjectively reduced the severity of the disease, thereby reducing the impact on HRQoL. [7, 9, 43]. Our result that the sense of loneliness has a negative impact on the quality of life highlights an important subjective aspect that can suggest fields of intervention and prevention.

The scientific literature highlight that other factors that may contribute to this decline of health are the decrease in economic resources illness widowhood and impaired mobility: all these factors may result in increased risk for social isolation In our sample no patient has economic problems but it is interesting to evaluate also the relation with this issue in further studies. 
In conclusion we can make the following considerations: the presence of a good perception of relationships absence of a sense of loneliness and its link with good quality of life can help a positive adaptation to the condition of chronic illness and multi-morbidity It can also promote quality of life by improving natural defenses.

The absence of a sense of the dimension of loneliness can be an indicator of patients with a good adaptation to disease conditions related to medical treatment.

Patients with a sense of loneliness should be subjected to closer surveillance than those without these experience. Early identification of these patients may be necessary to provide mental health professionals with the opportunity for proactive intervention (Masterson 2015). Other studies will further explore new methods to support the specific needs of patients including interpersonal and emotional needs in the context of holistic approach to psycho-physical health.

Current research has shown that a sense of loneliness is a common risk factor for quality of life in elderly patients with multimorbidity. The level of social support and other protective factors should be explored in future research especially in patients without self-sufficiency.

Limitations First all the subjects in this study were affected by determined pathology and care is needed when extrapolating these results to other diseases. Second a sampling bias was present in the data because all the subjects attended one institution and thus were not representative of elderly patients affected by diseases and multimorbidity in general. Third our results provide a snapshot of quality of life sense of loneliness and other variables after diagnosis and during the treatment phase. Although the results in QoL symptoms may differ during the treatment phase or at other points in the disease journey Quality of life may evolve naturally over time once patients begin treatment.

\section{Declarations}

\section{Ethics approval and consent to participate}

The patient gave his consent to publish his data and signed the consent form

Funding No funds were received for this study.

\section{Competing interests}

All the authors wish to confirm that there are no known conflicts of interest associated with this publication and there has been no financial support for this work that could have influenced its outcome.

\section{Authors' contributions}

Anna Vespa Ph.D.: Study planning, test administration, results analysis, article writing.

Maria Velia Giulietti Psy.D: Test administration, results analysis, article writing. 
Pisana Gattafoni, M.D.: Exams and clinical evaluation (T0,T1), results analysis

Roberta Spatuzzi Psy.D.: Results analysis and article writing.

Paolo Fabbietti, MSc: Statistical analysis including power of the sample.

Ethics approval and consent to participate

Ethics Committee Approval (INRCA_IRCCS- National Institute of Science and Health for Aging)

\section{References}

1. Vetrano DL, Foebel AD, Marengoni A, Brandi V, Collamati A, Heckman GA, et al. Chronic diseases and geriatric syndromes: the different weight of comorbidity. Eur J Intern Med. 2016;27:62-7.

2. Navickas R, Petric VK, Feigl AB, Seychell M. Multimorbidity: what do we know? What should we do? J Comorbidity. 2016;6:4-11.

3. Prakeh AK, Goodman RA, Gordon C, Koh HK. Managing multiple chronic conditions: a strategic framework for improving health outcomes and quality of life. Public Health Rep. 2011;126:460-71.

4. Barnett K, Mercer SW, Norbury M, Watt G, Wyke S, Guthrie B. Epidemiology of multimorbidityand implications for health care research and medical education: a cross-sectional study. Lancet. 2012;380:37-43.

5. Lehnert T, Heider D, Leicht $H$, Heinrich S, Corrieri S, Luppa M, et al. Review: health care utilization and costs of elderly persons with multiple chronic conditions. Med Care Res Rev. 2011;68:387-420.

6. Haro JM, Olaya B, Domènech-Abella J, Moneta MV, Lara E, Caballero FF, et al. All-cause mortality and multimorbidity in older adults: The role of social support and loneliness. Exp Gerontol. 2017;99:12026.

7. Arokiasamy P, Uttamacharya U, Jain K, Biritwum RB, Yawson AE, Wu F, et al. The impact of multimorbidity on adult physical and mental health in low- and middle-income countries: what does the study on global ageing and adult health. SAGE reveal? BMC Med 2015;13:178.

8. Violan C, Foguet-Boreu Q, Flores-Mateo G, Salisbury C, Blom J, Freitag M, et al. Prevalence determinants and patterns of multimorbidity in primary care: a systematic review of observational studies. PLoS One. 2014;9:e102149.

9. Salisbury C, Johnson L, Purdy SJ, Valderas M, Montgomery AA. Epidemiology and impact of multimorbidity in primary care: a retrospective cohort study. Br J Gen Pract. 2011;61:12-21.

10. Galenkamp H, Braam AW, Huisman M, Deeg DJ. Somatic multimorbidity and self-rated health in the older population. J Gerontol B Psychol Sci Soc Sci. 2011;66:380-86. 
11. Kadam UT, Croft PR. Clinical multimorbidityand physical function in older adults: a record and health status linkage study in general practice. Fam Pract. 2007;24:412-49.

12. Walker AE. Multiple chronic diseases and quality of life: patterns emerging from a large national sample Australia. Chronic IIIn. 2007;3:202-18.

13. Li J, Green M, Kearns B, Holding E, Smith C. Haywood Aet al. Patterns of multimorbidity and their association with health outcomes within Yorkshire England: baseline results from the Yorkshire Health Study. BMC Public Health. 2016;16:649.

14. Singer L, Green M, Rowe F, Ben-Shlomo Y, Morrissey K. Social determinants of multimorbidityand multiple functional limitations among the ageing population of England 2002-2015. SSM Popul Health. 2019;8:100413.

15. Luo Y, Hawkley LC, Waite LJ, Cacioppo JT. Loneliness health and mortality in old age: a national longitudinal study. Soc Sci Med. 2012;74:907-14.

16. Yarnall AJ, Sayer AA, Clegg A, Rockwood K, Parker S, Hindle JV. New horizons in multimorbidity in older adults. Age Ageing. 2017;466:882-88.

17. Quirk SE, Stuart AL, Berk M, Pasco JA, Brennan Olsen SL, Koivumaa-Honkanen H, et al. Personality disorder is an excess risk factor for physical multimorbidity among women with mental state disorders. Psychiatry Res. 2017;257:546-49.

18. Stubbs B, Vancampfort D, Veronese N, Kahl KG, Mitchell AJ, Lin PY, et al. Depression and physical health multimorbidity: primary data and country-wide meta-analysis of population data from 190 593 people across 43 low- and middle-income countries. Psychol Med. 2017;47:2107-17.

19. Kristensen $\mathrm{K}$, König HH, Hajek A. The longitudinal association of multimorbidity on loneliness and network size: Findings from a population-based study. Int J Geriatr Psychiatry. 2019;34:1490-97.

20. Perlman D, Peplau LA. Toward a social psychology of loneliness. In: Duck S, Gilmour R, editors. Personal relationships: Relationships in disorder. London: Academic Press; 1981. pp. 31-56.

21. Hawkley LC, Cacioppo JT. Loneliness matters: a theoretical and empirical review of consequences and mechanisms. Ann Behav Med. 2010;40:218-27.

22. Cohen-Mansfield J, Shmotkin D, Goldberg S. Loneliness in old age: longitudinal changes and their determinants in an Israeli sample. Int Psychogeriatr. 2009;21:1160-170.

23. Holt-Lunstad J, Smith TB, Baker M, Harris T, Stephenson D. Loneliness and social isolation as risk factors for mortality: a meta-analytic review. Perspect Psychol Sci. 2015;10:227-37.

24. Cacioppo JT, Hawkley LC. Perceived social isolation and cognition. Trends Cogn Sci. 2009;13:44754.

25. Courtin E, Knapp M. Social Isolation Loneliness and Health in Old Age: A Scoping Review. Health Soc Care Community. 2017;25:799-812.

26. Kuiper JS, Smidt N, Zuidema SU, Comijs HC, Oude Voshaar RC, Zuidersma M. A Longitudinal Study of the Impact of Social Network Size and Loneliness on Cognitive Performance in Depressed Older Adults. Aging Ment Health. 2020;24:889-97. 
27. Richard A, Rohrmann S, Vandeleur CL, Schmid M, Barth J, Eichholzer M. Loneliness is adversely associated with physical and mental health and lifestyle factors: Results from a Swiss national survey. PLoS One. 2017;12:e0181442.

28. Cantarero-Prieto D, Pascual-Sáez M, Blázquez-Fernández C. Social isolation and multiple chronic diseases after age 50: A European macro-regional analysis. PLoS One. 2018;13:e0205062.

29. Stickley A, Koyanagi A. Physical multimorbidity and loneliness: a population-based study. PLoS ONE. 2018;13:e0191651.

30. Domènech-Abella J, Lara E, Rubio-Valera M, Olaya B, Moneta MV, Rico-Uribe LA, et al. Loneliness and depression in the elderly: the role of social network. Soc Psychiatry Psychiatr Epidemiol. 2017;52:381-90.

31. Perissinotto CM, Stojacic Cenzer I, Covinsky KE. Loneliness in older persons: a predictor of functional decline and death. Arch Intern Med. 2012;17:1078-84.

32. The RO, Menzies OH, Connolly MJ, Doughty RN, Wilkinson TJ, Pillai AB, et al. Patterns of MultiMorbidity and Prediction of Hospitalisation and All-Cause Mortality in Advanced Age. Ageing. 2018;47:261-68.

33. Kawada T. Loneliness and Mortality in Older Men: Causal Association. Am J Geriatr Psychiatry. 2017;25:102.

34. Ong AD, Uchino BN, Wethington E. Loneliness and Health in Older Adults: A Mini-Review and Synthesis Gerontology. 2016;62:443-49.

35. Cornwell EY, Waite LJ. Social disconnectedness perceived isolation and health among older adults $J$ Health Soc Behav. 200950 31-48.

36. Taylor HO, Taylor RJ, Nguyen AW. \& Chatters L. Social Isolation Depression and Psychological Distress Among Older Adults. J Aging Health. 2018;30:229-46.

37. Musich S, Wang SS, Hawkins K, Yeh CS. The impact of loneliness on quality of life and patient satisfaction among older sicker adults. Gerontol Geriatr Med. 2015;1:2333721415582119.

38. Jessen MAB, Pallesen AVJ, Kriegbaum M, Kristiansen M. The Association Between Loneliness and Health - A Survey-Based Study Among Middle-Aged and Older Adults in Denmark. Aging Ment Health. 2018;22:1338-43.

39. Liebert MA, Snodgrass JJ, Naidoo N, Chatterji S, Kowal P, Yawson AE, et al. The impact of multimorbidity on adult physical and mental health in low- and middle-income countries: what does the study on global ageing and adult health SAGE reveal? BMC Med. 2015;13:178.

40. Wister A, Kendig H, Mitchell B, Fyffe I, Loh V. Multimorbidity health and aging in Canada and Australia: a tale of two countries. BMC Geriatr. 2016;16:163.

41. Bao XY, Xie YX, Zhang XX, Peng X, Huang JX, Du QF, Wang PX The association between multimorbidity and health-related quality of life: a cross-sectional survey among community middleaged and elderly residents in southern China Health Qual Life Outcomes 2019;171:107 doi: 101186/s12955-019-1175-0. 
42. Barlow MA, Liu SY, Wrosch C. Chronic illness and loneliness in older adulthood: the role of selfprotective control strategies. Health Psychol. 2015;34:870-79.

43. Berkman LF, Glass T, Brissette I, Seeman TE. From social integration to health: Durkheim in the new millennium. Soc Sci Med. 2000;51:843-57.

44. Mlinac ME, Feng MC. Assessment of Activities of Daily Living Self-Care and Independence. Arch Clin Neuropsychol. 2016;31:506-16.

45. Ustun TB, Chatterji S, Kostanjsek N, Rehm J, Kennedy C, Epping-Jordan J, Saxena S, von Korff M, Pull C. WHO / NIH Joint Project Developing the World Health Organization Disability Assessment Schedule. Bull World Health Organ. 2010;88:815-23.

46. Magni E, Binetti G, Bianchetti A, Rozzini R, Trabucchi M. Mini-Mental State Examination: A Normative Study in Italian Elderly Population. Eur J Neurol. 1996;3:198-202.

47. Musicco M, Sorbi S, Bonavita V, Caltagirone C. Study Group on Validation of the Guidelines for the Diagnosis of Dementia and Alzheimer's Disease of the Italian Neurological Society Validation of the Guidelines for the Diagnosis of Dementia and Alzheimer's Disease of the Italian Neurological Society Study in 72 Italian neurological centers and 1549 patients. Neurol Sci. 2004;25:289-95.

48. Di Bella O, Cocchiara RA, De Luca A, Frusone F, Aceti V, Sestili C, D'Egidio V, Mannocci A, Monti M, La Torre G. Functional Assessment of Cancer Therapy Questionnaire for Breast Cancer (FACT-B + 4): Italian version validation. Clin Ter. 2018;169:e151-4.

49. De Jong Gierveld J, Van Tilburg T. The De Jong Gierveld. short scales for emotional and social Ioneliness: tested on data from 7 countries in the UN generations and gender surveys. Eur $\mathrm{J}$ Ageing. 2010;7:121-30.

50. De Girolamo G 1, Rucci P, Scocco P, Becchi A, Coppa F, D'Addario A, Darú E, Leo DD, Galassi L, Mangelli L, Marson C, Neri G, Soldani L. Quality of Life Assessment: Validation of the Italian Version of the WHOQOL-Brief. Epidemiol Psichiatr Soc. 2000;9:45-55.

\section{Tables}


Table 1. Characteristics of sample

\begin{tabular}{|c|c|}
\hline Variables & $\mathrm{N}=162$ \\
\hline Age (years) & $71.6 \pm 5.5$ \\
\hline Gender (female) & $115(71.0)$ \\
\hline \multicolumn{2}{|l|}{ Marital status } \\
\hline Single & $25(15.4)$ \\
\hline Married & $65(40.1)$ \\
\hline Widow & $64(39.5)$ \\
\hline Separated/Divorced & $8(4.9)$ \\
\hline Hypertension & $126(77.8)$ \\
\hline Diabetes & $49(30.2)$ \\
\hline Ictus & $31(19.1)$ \\
\hline Cancer & $19(11.7)$ \\
\hline Other pathologies & $89(54.9)$ \\
\hline Multimorbidity (at least 3 pathologies & 105 (64.8) \\
\hline
\end{tabular}

Data are expressed by mean \pm standard deviation for continuous variables, and by $n$ (\%) for categorical

Table 2. Independent samples's test

\begin{tabular}{|l|c|c|} 
& Variables & p \\
\hline PWB & 3.074 & 0.003 \\
\hline SWB & 0.341 & 0.003 \\
\hline EWB & 1.36 & 0.012 \\
\hline FWB & 5.954 & 0.001 \\
\hline
\end{tabular}

Physical Well-Being (PWB); Social Well-Being(SWB); Emotional Well-Being(EWB); Functional Well-Being (FWB) 
Table 3. Coefficients (dependent variable: PWB)

\begin{tabular}{l|c|c|c|c|}
\hline & \multirow{2}{*}{ P } & & \multicolumn{2}{|c|}{$95.0 \%$ CI } \\
\cline { 4 - 5 } & & $\mathbf{p}$ & Lower & Upper \\
\hline (Constant) & 4.94 & $<0.001$ & 3.366 & 6.514 \\
\hline MULTIMORBIDITY & -0.154 & 0.169 & -0.374 & 0.066 \\
\hline AGE & -0.024 & 0.014 & -0.043 & -0.005 \\
\hline GENDER & -0.134 & 0.228 & -0.353 & 0.085 \\
\hline SENSE OF LONELINESS & -0.435 & $<0.001$ & -0.575 & -0.295 \\
& & & & \\
\hline \multirow{2}{*}{ WHOQOL AGE } & 0.933 & $<0.001$ & 0.659 & 1.208 \\
\cline { 4 - 5 }
\end{tabular}

Physical Well-Being (PWB)

\begin{tabular}{|l|c|c|c|c|}
\hline \multicolumn{5}{|c|}{ Table 4. Coefficients (dependent variable: SWB) } \\
\hline & & & \multicolumn{2}{|c|}{$95.0 \%$ CI } \\
\cline { 4 - 6 } & $\mathbf{B}$ & $\mathbf{p}$ & Lower & Upper \\
\hline (Constant) & 4.7 & $<0.001$ & 2.895 & 6.506 \\
\hline MULTIMORBIDITY & -0.338 & 0.009 & -0.589 & -0.088 \\
\hline AGE & -0.022 & 0.051 & -0.045 & 0 \\
\hline SENDER & -0.006 & 0.956 & -0.238 & 0.225 \\
\hline WHOQOL AGE & -0.401 & $<0.001$ & -0.55 & -0.253 \\
& & & & \\
\cline { 4 - 6 } & 0.522 & 0.001 & 0.226 & 0.817 \\
\hline
\end{tabular}

Social Well-Being (SWB)

\begin{tabular}{l|c|c|c|c|}
\hline \multicolumn{5}{|c|}{ Table 5. Coefficients (dependent variable: EWB) } \\
\hline & & & \multicolumn{2}{|c|}{$95.0 \%$ CI } \\
\cline { 4 - 6 } & $\mathbf{B}$ & $\mathbf{p}$ & Lower & Upper \\
\hline (Constant) & 3.25 & $<0.001$ & 1.515 & 4.984 \\
\hline MULTIMORBIDITY & -0.063 & 0.604 & -0.302 & 0.176 \\
\hline AGE & -0.001 & 0.939 & -0.022 & 0.02 \\
\hline GENDER & -0.054 & 0.652 & -0.292 & 0.183 \\
\hline WENSE OF LONELINESS & -0.494 & $<0.001$ & -0.646 & -0.343 \\
& & & & \\
\hline
\end{tabular}

Emotional Well-Being (EWB) 


\begin{tabular}{|c|c|c|c|c|}
\hline & & & \multicolumn{2}{|c|}{$95.0 \% \mathrm{CI}$} \\
\hline & B & p & Lower & Upper \\
\hline (Constant) & 4.556 & $<0.001$ & 3.072 & 6.04 \\
\hline MULTIMORBIDITY & -0.268 & 0.012 & -0.475 & -0.06 \\
\hline AGE & -0.023 & 0.013 & -0.041 & -0.005 \\
\hline GENDER & -0.001 & 0.991 & -0.208 & 0.205 \\
\hline SENSE OF LONELINESS & -0.429 & $<0.001$ & -0.562 & -0.297 \\
\hline WHOQOL AGE & 0.703 & $<0.001$ & 0.444 & 0.962 \\
\hline
\end{tabular}

Functional Well-Being (FWB) 